\title{
ESTUDO DAS CONDIÇÕES DE SAÚDE DAS CRIANÇAS DO MUNICÍPIO DE SÃO PAULO, SP (BRASIL), 1984/1985
}

\author{
$X$. CONSUMO ALIMENTAR*
}

\author{
Sophia Cornbluth Szarfarc** \\ Carlos Augusto Monteiro** \\ Mauricio Meyer** \\ Eliete Salomon Tudisco*** \\ Isildinha Marques dos Reis****
}

SZARFARC, S.C. et al. Estudo das condições de saúde das crianças do Município de São Paulo, SP

(Brasil), 1984/1985. X - Consumo Alimentar. Rev. Saúde públ., S. Paulo, 22:266-72, 1988.

\begin{abstract}
RESUMO: Como parte de amplo estudo epidemiológico sobre condiçðes de saúde na infância, uma amostra probabilística de menores de cinco anos residentes no Município de São Paulo $(n=305)$ foi estudada com relação à adequação nutricional de suas dietas. Através do inquérito recordatório do consumo alimentar nas últimas 24 horas evidenciou-se que: a) a partir de um ano de idade, as dietas tornam-se insuficientes para cobrir as necessidades energéticas de grande parte da população; b) em todas as idades, mas particularmente nos primeiros dois anos de vida, o aporte dietético de ferro está muito abaixo das quantidades recomendadas para o consumo do nutriente; $\mathrm{c}$ ) em todas as idades, o aporte de proteina e de vitamina $\mathrm{A}$ alcança valores satisfatórios. Tais achados mostraram-se compativeis com a avaliação clínico-laboratorial do estado nutricional realizada simultaneamente ao inquérito alimentar. A estratificaçăo social da amostra revelou que o nivel sócio-econômico familiar influencia fortemente o consumo energético e, de forma menos intensa, o consumo de ferro. Na discussão dos prováveis fatores responsáveis pelos déficits dietéticos encontrados, hipóteses distintas são aventadas para o déficit energético e para o déficit de ferro. No caso do déficit energético, a origem básica do problema pareceria residir no baixo poder aquisitivo da população que condiciona insuficiente disponibilidade de alimentos e ingestão quantitativamente deficiente. No caso do déficit de ferro, a mesma hipótese valeria inteiramente apenas a partir da idade de dois anos. Antes desta idade, fatores relacionados ao desmame precoce e ao atraso na introdução de alimentos ricos em ferro aparentemente seriam mais importantes.
\end{abstract}

UNITERMOS: Saúde da criança. Consumo de alimentos. Inquéritos nutricionais. Nutrição infantil. Distúrbios da nutrição infantil.

\section{INTRODUÇÃO}

A inclusão do estudo do consumo alimentar em inquéritos destinados a estabelecer condições de saúde é fato que dispensa justificaçôes. Ainda mais necessárias se torna a referida inclusão quando o segmento populacional de interesse é particularmente vulnerável a deficiências nutricionais, como é o caso da população de menores de cinco anos 12 .

Embora importantes, são escassas em nosso meio as informações existentes sobre consumo alimentar. A nível nacional, os dados disponiveis procedem de inquérito realizado pela Fundação IBGE há mais de uma década (1974/75), com o incoveniente de que as informações fornecidas pela mesma, dizem respeito ao consumo médio de unidades familiares e não ao consumo de indivíduos ${ }^{8}$. A nível de regiões ou municipios, as informações existentes provêm em geral de pequenos estudos realizados em amostras com base populacional pouco definida e de difícil inferência para conjuntos maiores da população. Uma fonte alternativa de informações, freqüentemente utilizada, são os inquéritos domiciliares de despesa familiar conduzidos por instituições que medem custo de vida ${ }^{3,7,13}$. Tais inquéritos apresentam, entretanto, duas grandes limitações: a) deduzem a ingestão alimentar a partir das compras e estoques de ali-

\footnotetext{
* Pesquisa financiada pela Financiadora de Estudos e Projetos - FINEP (Convênio 4.1.83.0698.00) e Fundação de Amparo à Pesquisa do Estado de São Paulo (Processo n: 86/0392-7)

** Departamento de Nutrição da Faculdade de Saúde Pública da Universidade de São Paulo - Av. Dr. Arnaldo, 715 01255 - São Paulo, SP - Brasil.

*** Departamento de Medicina Preventiva da Escola Paulista de Medicina - Rua Botucatu, 720 - 04023 - São Paulo, SP - Brasil.

**** Departamento de Epidemiologia da Faculdade de Saúde Pública da Universidade de São Paulo - Av. Dr. Arnaldo, 715 - 01255 - São Paulo, SP - Brasil.
} 
mento no domicílio; e b) não consideram as diversidades de consumo existentes dentro da unidade familiar.

Complexidades metodológicas aliadas a questões de custo e de falta de pessoal qualificado poderiam explicar em parte a escassez de informações sobre consumo alimentar em nosso meio. De fato, estudos de consumo implicam diferentes ordens de dificuldades, incluindo as relacionadas: à aferição precisa da ingestão alimentar; à transformação da ingestão em quantidades exatas de nutrientes; e à escolha e interpretação de indicadores de adequação. Embora diferentes técnicas e instrumentos tenham sido desenvolvidos para cada uma destas etapas, as alternativas disponíveis estão longe de serem satisfatórias, fornecendo, de modo geral, precisão inferior àquela desejada.

Entre abril de 1984 e junho de 1985 uma amostra probabilística da população de menores de 5 anos residente no Município de São Paulo foi estudada objetivando-se compreender diferentes aspectos relacionados às suas condições de saúde. Em artigos anteriores desta série foram apresentados resultados envolvendo o quadro de morbidade, o estado nutricional e a assistência recebida pelas crianças do Município. No presente artigo são apresentados os resultados referentes ao módulo do estudo que examinou a adequação nutricional das dietas consumidas pela população.

\section{METODOLOGIA}

A população de estudo corresponde a 305 crianças de zero a 59 meses de idade, residentes no Município de São Paulo constituindo sub-amostra aleatória da casuística total $(n=1016)$, estudada pela pesquisa "Estudo das condiçoes de saúde das crianças do Município de São Paulo"16. O processo completo de amostragem está descrito em trabalho anterior $^{16}$, onde se evidencia o caráter probabilístico dos procedimentos adotados no sorteio das crianças estudadas. $O$ consumo alimentar da criança foi estabelecido através da aplicação de inquérito recordatório relativo ao consumo das últimas 24 horas*. O inquérito foi aplicado junto às mães ou responsáveis pelas crianças, sendo executado por médicos pediatras treinados e supervisionados por nutricionistas experientes no método de inquérito utilizado. No caso específico do consumo de leite materno, as quanti- dades ingeridas foram estimadas de acordo com valores padronizados ${ }^{2}$.

A transformação dos alimentos ingeridos em energia e nutrientes foi realizada com base na Tabela de Composição de Alimentos compilada pela Fundação IBGE ${ }^{9}$.

A adequação nutricional das dietas foi analisada com relação a energia, proteína, ferro e vitamina A. A escolha desses elementos da dieta decorreu de sua relação com as deficiências nutricionais de maior relevância epidemiológica em nosso meio ${ }^{1,21}$.

As adequações energética e protéica das dietas foram analisadas tendo por base os valores de referência propostos pelo Comitê Misto de Especialistas $\mathrm{FAO} / \mathrm{WHO} / \mathrm{ONU}^{4}$. Em ambos os casos foram calculados os seguintes indicadores:

- adequação percentual média das dietas, ou seja, a média das adequaçðes percentuais individuais entre o consumo observado e o consumo esperado (da energia e de proteína), sendo o consumo esperado aquele indicado pelos valores de referência $\mathrm{FAO} /$ WHO/ONU para crianças com peso equivalente ao percentil 50 para idade e sexo (padrão antropométrico National Center for Health Statistics (NCHS) ${ }^{10}$ );

- percentagem de dietas deficientes, ou seja, dietas que ficam aquém dos valores de referência indicados para crianças com peso equivalente ao percentil 3 ;

- percentagem de dietas que atingem as recomendações, ou seja, dietas que igualam ou excedem os valores de referência indicados para crianças com peso equivalente ao percentil 50, no caso de energia, e com peso equivalente ao percentil 97 , no caso de proteína.

As adequações do consumo de ferro e de vitamina A foram analisadas partindo-se das recomendações propostas para a população brasileira por Martins ${ }^{15}$. Como valores de recomendação, os de consumo propostos por Martins ${ }^{15}$, visam a cobrir as necessidades de praticamente todos os indivíduos da população, incluindo-se aqueles cujas necessidades situem-se até dois desvios-padrão acima da média populacional. Tendo em conta este fato, no caso dos indicadores adequação percentual média das dietas e percentagem de dietas deficientes, os valores de referência utilizados são os

\footnotetext{
* A escolha recaiu neste método por ser de fácil e rápida aplicação e por permitir a avaliação de consumo dos nutrientes de interesse da pesquisa com a precisão necessária ${ }^{5}, 6,14$. Pelo método recordatório, a quantidade consumida de alimentos é estimada a partir das medidas caseiras utilizadas pelas mães (frascos, copos, colheres, e outras) e correspondência previamente padronizada, entre medidas caseiras e peso dos alimentos.
} 
valores originais de Martins's subtraidos, respectivamente, de dois e de quatro desviospadrão*. No caso do indicador percentagem de dietas que atingem as recomendaçôes, os valores de referência adotados naturalmente coincidem com os valores propostos por Martins ${ }^{15}$.

As dietas que não são classificadas como "deficientes" e nem atendem as recomendaçðes estabelecidas devem ser interpretadas como dietas "de risco".

A adequação da dieta foi estudada com relação à faixa etária da criança e ao estrato sócio-econômico da família. Como nos demais artigos desta série, utilizou-se a escolaridade do chefe da familia como indicador de nível sócio-econômico, correspondendo os seguintes estratos aos seguintes níveis de escolaridade: I $=$ superior, $\mathrm{II}=$ colegial, $\mathrm{III}=$ ginasial, $\mathrm{IV}=$ primária, $\mathrm{V}=$ primária incompleta, $\mathrm{VI}=\mathrm{au}-$ sência de escolaridade**. No presente artigo, devido à utilização de sub-amostra, foram reunidos em uma só categoria os estratos I, II e III, o mesmo se fazendo com estratos V e VI.

\section{RESULTADOS}

A Tabela 1 apresenta o consumo médio diário de energia e nutrientes nas diversas faixas etárias da população. O consumo de energia, proteína e ferro aumenta com a idade até os 24 meses, pouco variando após esta idade. $O$ consumo de vitamina $\mathrm{A}$ alcança maiores valores no primeiro ano de vida, caindo sensivelmente nas demais idades.

A adequação nutricional média das dietas é apresentada na Tabela 2 . Com relação a proteína e vitamina $A$, as adequaçðes médias si- tuam-se sempre bem acima de $100 \%$, sugerindo consumo satisfatório destes nutrientes. A adequação média do consumo energético ultrapas. sa $100 \%$ apenas no primeiro ano de vida, tornando-se progressivamente inferior àquele valor nas idades subseqüentes. Com referência a ferro, as adequações médias nunca alcançam $100 \%$, sendo especialmente baixas no primeiro ano de vida.

O total das dietas deficientes em energia somou $40,0 \%$, observando-se que em apenas $31,2 \%$ dos casos as recomendaçð̃es energéticas foram atingidas (Tabela 3). Ambos os índices revelam situação privilegiada do consumo energético no primeiro ano de vida e tendência à piora nas idades subseqüentes.

Com relação a proteína; são desprezíveis as proporções de dietas deficientes, observando-se que na maioria dos casos a ingestão é superior às recomendações (Tabela 4).

A percentagem de dietas deficientes em ferro foi de $48,0 \%$, notando-se que em apenas $13,7 \%$ dos casos as recomendações para o nutriente foram atingidas (Tabela 5). Digna de nota é a situação encontrada no primeiro ano de vida, onde nenhuma dieta atingiu a recomendação da ingestão.

Com referência à vitamina $A$, a proporção de dietas deficientes foi relativamente pequena, $10,5 \%$, sendo de $70,8 \%$ a proporção de casos que atenderam a recomendação (Tabela 6). Vale destacar neste caso a virtual ausência de dietas deficientes em vitamina A no primeiro ano de vida.

A Tabela 7 apresenta a variação da frequêencia de dietas deficientes conforme o nível sócio-econômico da população. Com relação à energia, observa-se nítido aumento da freqüên-

TABELA 1

Consumo médio diário de energia, proteína, vitamina A e ferro entre crianças de zero a 59 meses do Município de São Paulo (1984/85)

\begin{tabular}{|c|c|c|c|c|c|}
\hline $\begin{array}{l}\text { Faixa } \\
\text { Etária } \\
\text { (meses) }\end{array}$ & $N^{*}$ & $\begin{array}{l}\text { Energia } \\
(\text { Kcal }) \\
\begin{array}{ll}\mathbf{x} & (\mathrm{s})\end{array}\end{array}$ & $\begin{array}{l}\text { Proteína } \\
\bar{x}^{(g)}(\mathrm{s})\end{array}$ & $\begin{aligned} & \text { Vit. A } \\
&(\mathrm{Eq} . \\
& \overline{\mathrm{x}} \begin{array}{l}\text { Ret) } \\
(\mathrm{s})\end{array}\end{aligned}$ & $\begin{array}{l}\text { Ferro } \\
(\mathrm{mg}) \\
\bar{x}^{(\mathrm{s})}\end{array}$ \\
\hline $\begin{array}{c}0 \vdash 6 \\
6 \vdash 12 \\
12 \vdash 24 \\
24 \vdash 36 \\
36 \vdash 48 \\
48 \vdash 60\end{array}$ & $\begin{array}{l}33 \\
25 \\
67 \\
68 \\
59 \\
53\end{array}$ & $\begin{array}{r}718(334) \\
930(338) \\
1052(391) \\
1166(567) \\
1178(455) \\
1150(455)\end{array}$ & $\begin{array}{l}23,6(15,7) \\
34,9(12,5) \\
47,1(52,4) \\
48,7(33,3) \\
46,1(18,9) \\
43,3(20,1)\end{array}$ & $\begin{array}{c}908(729) \\
1262(2065) \\
568(709) \\
590(1059) \\
448(315) \\
538(554)\end{array}$ & $\begin{array}{l}1,80(1,41) \\
4,72(2,42) \\
6,08(3,69) \\
6,80(4,34) \\
7,24(3,23) \\
6,96(3,93)\end{array}$ \\
\hline
\end{tabular}

$* \mathrm{~N}=$ Número de crianças estudadas.

* Para se estabelecer o desvio-padrão das necessidades em ferro e em vitamina A, considerou-se que o coeficiente de variação daquelas necessidades é idêntico ao aceito para energia e proteína, ou seja, $15 \%{ }^{4}$.

** Em relação à terminologia atualmente utilizada, os cursos primário, ginasial e colegial correspondem, respectivamente, às quatro primeiras séries do primeiro grau, às quatro últimas séries do primeiro grau e às três séries do segundo grau. 
TABELA 2

Adequação percentual média do consumo de energia, proteína, ferro e vitamina A entre crianças de zero a 59 meses de idade do Município de São Paulo (1984/85)

\begin{tabular}{llllll}
\hline $\begin{array}{l}\text { Faixa } \\
\begin{array}{l}\text { Etária } \\
\text { (meses) }\end{array}\end{array}$ & $\mathrm{N}$ & Energia & Proteína & Ferro & Vitamina A \\
\hline $0 \vdash 6$ & 33 & 133,2 & 234,6 & 40,8 & 414,1 \\
$6 \vdash 12$ & 25 & 111,1 & 304,0 & 78,9 & 287,0 \\
$1 \vdash 24$ & 67 & 90,0 & 301,2 & 88,2 & 238,3 \\
$24 \vdash 36$ & 68 & 85,4 & 275,2 & 94,1 & 243,0 \\
$36 \vdash 48$ & 59 & 78,2 & 234,9 & 90,8 & 234,1 \\
$48 \vdash 60$ & 53 & 71,2 & 271,8 & 79,1 & 274,0 \\
\hline Total & 305 & 89,8 & 270,4 & 82,5 & 2 \\
\hline
\end{tabular}

TABELA 3

Indicadores da adequação do consumo energético entre crianças de zero a 59 meses de idade do Município de São Paulo (1984/85)

\begin{tabular}{lccc}
\hline $\begin{array}{l}\text { Faixa } \\
\text { Etária } \\
\text { (meses) }\end{array}$ & $N$ & $\begin{array}{c}\text { \% de Dietas } \\
\text { Deficientes } \\
\text { em Energia* }\end{array}$ & $\begin{array}{c}\text { \% de Dietas que } \\
\text { Atingem a } \\
\text { Recomendaçâo } \\
\text { Energética* }\end{array}$ \\
\hline $0 \vdash 6$ & 33 & 12,1 & 63,7 \\
$6 \vdash 12$ & 25 & 4,0 & 56,0 \\
$12 \vdash 24$ & 67 & 37,3 & 34,4 \\
$24 \vdash 36$ & 68 & 54,4 & 25,0 \\
$36 \vdash 48$ & 59 & 49,1 & 20,4 \\
$48 \vdash 60$ & 53 & 49,0 & 15,2 \\
\hline Total & 305 & 40,0 & 31,2 \\
\hline
\end{tabular}

* Conforme definidas na metodologia. As dietas não "deficientes", mas que estão aquém das recomendaçōes, corresponderam ao que se pode denominar "dietas de risco".

\section{TABELA 5}

Indicadores de adequação de consumo de ferro entre crianças de zero a 59 meses de idade do Município de São Paulo (1984/85)

\begin{tabular}{lccc}
\hline $\begin{array}{l}\text { Faixa } \\
\begin{array}{l}\text { Etária } \\
\text { (meses) }\end{array}\end{array}$ & $\mathrm{N}$ & $\begin{array}{c}\text { \% de Dietas } \\
\text { Deficientes } \\
\text { em Ferro* }\end{array}$ & $\begin{array}{c}\text { \% de Dietas que } \\
\text { Atingem a } \\
\text { Recomendação } \\
\text { em Ferro* }\end{array}$ \\
\hline $0 \vdash 6$ & 33 & 97,0 & 0,0 \\
$6 \vdash 12$ & 25 & 60,0 & 0,0 \\
$12 \vdash 24$ & 67 & 48,5 & 16,7 \\
$24 \vdash 36$ & 68 & 42,4 & 13,7 \\
$36 \vdash 48$ & 59 & 33,9 & 13,6 \\
$48 \vdash 60$ & 53 & 38,3 & 14,9 \\
\hline Total & 305 & 48,0 & 13,7 \\
\hline
\end{tabular}

* Conforme definidas na metodologia. As dietas não "deficientes", mas que estão aquém das recomendaçōes, corresponderam ao que se pode denominar "dietas de risco".

\section{TABELA 4}

Indicadores de adequação do consumo de proteína entre crianças de zero a 59 meses de idade do Município de São Paulo (1984/85)

\begin{tabular}{lccc}
\hline $\begin{array}{l}\text { Faixa } \\
\begin{array}{l}\text { Etária } \\
\text { (meses) }\end{array}\end{array}$ & N & $\begin{array}{c}\text { \% de Dietas } \\
\text { Deficientes } \\
\text { em Proteínas }\end{array}$ & $\begin{array}{c}\text { \% de Dietas que } \\
\text { Atingem a } \\
\text { Recomendação } \\
\text { Protéica* }\end{array}$ \\
\hline $0 \vdash 6$ & 33 & 6,1 & 60,6 \\
$6 \vdash 12$ & 25 & 4,0 & 96,0 \\
$12 \vdash 24$ & 57 & 4,5 & 95,5 \\
$24 \vdash 36$ & 68 & 0,0 & 98,5 \\
$36 \vdash 48$ & 59 & 5,1 & 93,2 \\
$48 \vdash 60$ & 53 & 0,0 & 86,8 \\
\hline Total & 305 & 3,0 & 90,1 \\
\hline
\end{tabular}

* Conforme definidas na metodologia. As dietas não "deficientes", mas que estão aquém das recomendações, corresponderam ao que se pode denominar "dietas de risco".

\section{TABELA 6}

Indicadores de adequação do consumo de vitamina $A$ entre crianças de zero a 59 meses de idade do Município de São Paulo (1984/85)

\begin{tabular}{lccc}
\hline $\begin{array}{l}\text { Faixa } \\
\begin{array}{l}\text { Etária } \\
\text { (meses) }\end{array}\end{array}$ & N & $\begin{array}{c}\text { \% de Dietas } \\
\text { Deficientes } \\
\text { em Vitamina A* }\end{array}$ & $\begin{array}{c}\text { \% de Dietas que } \\
\text { Atingem a } \\
\text { Recomendação } \\
\text { em Vitamina A* }\end{array}$ \\
\hline $0 \vdash 6$ & 33 & 3,0 & 75,8 \\
$6 \vdash 12$ & 25 & 0,0 & 76,0 \\
$12 \vdash 24$ & 67 & 11,9 & 73,2 \\
$24 \vdash 36$ & 68 & 13,2 & 67,7 \\
$36 \vdash 48$ & 59 & 11,9 & 67,8 \\
$48 \vdash 60$ & 53 & 13,2 & 69,8 \\
\hline Total & 305 & 10,5 & 70,8 \\
\hline
\end{tabular}

* Conforme definidas na metodologia. As dietas não "deficientes", mas que estão aquém das recomendações, corresponderam ao que se pode denominar "dietas de risco". 
TABELA 7

Distribuição percentual de dietas deficientes entre crianças menores de cinco anos segundo estrato sócioeconômico. Município de São Paulo (1984/85)

\begin{tabular}{lccccc}
\hline \multirow{2}{*}{$\begin{array}{l}\text { Estrato } \\
\text { Sócio- } \\
\text { Econômico* }\end{array}$} & $N^{* *}$ & \multicolumn{4}{c}{ \% de Dietas Deficientes } \\
\cline { 3 - 6 } & & Energia & Proteína & Ferro & Vitamina A \\
\hline I / II / III & 86 & 27,9 & 3,5 & 39,5 & 7,0 \\
IV & 103 & 39,8 & 1,9 & 55,3 & 7,8 \\
V / VI & 113 & 48,7 & 3,5 & 50,4 & 15,9 \\
\hline Total & 302 & 39,7 & 3,0 & 49,0 & 10,6 \\
\hline
\end{tabular}

* Estratos sócio-econômicos constituídos a partir do nível de escolaridade do chefe da família da criança: $I=$ superior; II = colegial; III = ginasial $; \mathrm{IV}=$ primária completa $; \mathrm{V}=$ primária incompleta; $\mathrm{VI}=$ ausência de escolaridade.

** Três crianças não puderam ser classificadas quanto ao estrato sócio-econômico.

cia de dietas deficientes à medida que se passa dos estratos de melhor nível para os de pior nível sócio-econômico. Tendências semelhantes, ainda que menos claras, são observadas com relação ao consumo de ferro e vitamina $\mathrm{A}$. Com referência à proteina, as exíguas proporções de dietas deficientes não parecem guardar relação com o nível sócio-econômico.

\section{COMENTÁRIOS}

Através do estudo de amostra probabilistica das crianças menores de cinco anos residentes no Município de São Paulo, observou-se que:

1. a partir de um ano de idade as dietas tornam-se insuficientes para cobrir as necessidades energéticas de grande parte da população;

2. em todas as idades, mas particularmente nos primeiros dois anos de vida, o aporte dietético de ferro está abaixo das quantidades recomendadas para o consumo do nutriente;

3. em todas as idades, o aporte de proteína e de vitamina $A$ alcança valores satisfatórios;

4. as condiçð̃es sócio-econômicas da população condicionam, em maior grau, o consumo energético e, em menor grau, o consumo de ferro.

Considerando o grau de imprecisão a que estão sujeitas as avaliações nutricionais do consumo alimentar, convém examinar antes de mais nada a consistência dos achados dietéticos obtidos*.

Uma alternativa privilegiada na análise de consistência de achados dietéticos é a contrapo- sição dos mesmos a indicadores clínico-laboratoriais do estado nutricional.

Com relação a energia e ferro, pode-se dizer que os resultados da avaliação dietética coincidem com o quadro revelado pela avaliação do estado nutricional. Em publicaçðes anteriores desta série ${ }^{17}, 19$, baseadas na amostra integral da pesquisa "Estudo das condiçōes de saúde das crianças do município; evidenciou-se que: a) a desnutrição protéico-energética (DPE) e a anemia ocorrem com frequeência no Município de São Paulo; b) as crianças menores de um ano se apresentam protegidas da DPE; c) a anemia ocorre em todas as idades, mas sobretudo nos dois primeiros anos de vida; d) tanto a frequiência da DPE quanto da anemia são condicionadas pelo nível sócio-econômico da população, émbora tal condicionamento seja particularmente intenso apenas no caso da DPE.

$A$ avaliação do estado nutricional da população com relação à vitamina $\mathrm{A}$ foi feita na mencionada pesquisa apenas através da procura de lesð̃es oculares decorrentes da deficiência vitamínica, não sendo realizados exames bioquími$\cos$ da concentração plasmática do nutriente. Em nenhuma criança foram detectados sinais sugestivos da presença de deficiência de vitamina $A$, fato que, de certo modo, concorda com a pequena expressão da deficiência alimentar do nutriente.

Com relação a proteína, deve-se notar que a adequação satisfatória das dietas não colide com a ocorrência freqüente de DPE na população; revela apenas o predomínio do componente energético na origem do problema, fato, aliás, já verificado no inquérito nacional sobre

* A imprecisão das avaliaçães do consumo alimentar é caracteristica apenas do método recordatório a todas as técnicas de inquérito alimentar. A pesagem sistemática de todos os alimentos da dieta se, de um lado, melhora a precisão das quantidades efetivamente consumidas, de outro aumenta a probabilidade de modificaçðes na prática alimentar rotineira do individuo. 
consumo alimentar realizado pelo IBGE em $1974 / 75^{22}$.

Uma vez que o quadro dietético apontado pela avaliação alimentar aparenta ser razoavelmente consistente, torna-se lícito levantar hipóteses sobre os fatores responsáveis pelas deficiências encontradas e aventar eventuais recomendaçōes a seu controle.

Com relação ao déficit energético, a evidente relação com o nível sócio-econômico das famílias conduz a discussão basicamente para o lado de fatores sócio-econômicos, sobretudo aqueles relacionados ao poder aquisitivo da população. Cerca de dois terços das crianças amostradas pelo estudo pertencem a famílias que apresentavam renda inferior a um salário mínimo per capita, que é o nível mínimo de renda calculado como necessário para satisfazer as necessidades básicas de uma família residente no Município de São Paulo' ${ }^{16}$. Igualmente deve-se mencionar que no âmbito da amostra estudada são enormes as disparidades de renda: enquanto os $10 \%$ das famílias mais ricas retêm $41,2 \%$ do total de renda de todas as famílias, os $40 \%$ mais pobres ficam apenas com $12,0 \%$ da renda ${ }^{16}$.

Com relação ao déficit de ferro, a discussão sobre determinantes deve distinguir a situação encontrada nos dois primeiros anos de vida da situação encontrada nas idades subseqüentes. A partir dos dois anos, o déficit de ferro não ultrapassa o déficit energético da dieta, o que indica que a deficiência do mineral é secundária ao déficit quantitativo da dieta e, portanto, passível da mesma determinação sócio-econômica aventada para a deficiência energética. Antes daquela idade, sobretudo no primeiro ano de vida, verifica-se que o déficit de ferro ultrapassa de muito o déficit energético da dieta, sugerindo que a origem principal do problema estaria na composição e não propriamente na quantidade da dieta ingerida.

Observando-se a pauta alimentar das crianças nos dois primeiros anos de vida, verifica-se que, de fato, são escassas as fontes dietéticas de ferro. O leite materno, fonte de ferro de alta biodisponibilidade ${ }^{11}$, é ofercido para a maioria das crianças apenas até o terceiro ou quarto mês de vida ${ }^{18}$; após esta idade, é substituído pelo leite de vaca, o qual, diferentemente do leite materno, não aporta à criança ferro de fácil absorção. A carne aparece de modo sistemático na dieta infantil apenas depois de um ano de idade e, ainda assim, restrita a uma parte das crianças (apenas 53,2\% delas, de um a dois anos, referiram consumo de carne no dia anterior). O feijão, que poderia ser uma importante fonte alternativa de ferro, também entra na dieta infantil, de modo sistemático, apenas depois de um ano de idade e, ainda assim, em quantidades pequenas (o consumo médio de feijão por criança na idade de um a dois anos foi de $10,0 \mathrm{~g}$ por dia). Hortaliças de folha não fazem parte da alimentação habitual das crianças de nenhuma idade.

Uma vez que a composição da dieta, e não a quantidade da alimentação, é o elemento que parece condicionar a deficiência de ferro nos primeiros dois anos de vida, ações de caráter educativo, neste caso, não podem ser desconsideradas. Em particular, ações que possibilitem uma maior extensão do período de aleitamento materno e uma introdução mais precoce de alimentos ricos em ferro afiguram-se bastante promissora.

Os aspectos positivos encontrados na avaliação alimentar quanto a proteína e vitamina $\mathbf{A}$ devem ser creditados majoritariamente ao consumo de leite observado na amostra: a grande maioria das crianças $(90,6 \%)$ referiu consumo sistemático de leite, situando-se entre 400 e 500 $\mathrm{ml}$ a quantidade média ingerida por criança. Em artigo anterior ${ }^{20}$, evidencia-se a importância dos programas governamentais de distribuição de leite na explicação do consumo satisfatório do alimento, pela população.

\section{AGRADECIMENTOS}

À Marcia Regina Manfredini e à Maria Silvia Dantas Carvalho pelo trabalho dedicado de supervisão, revisão e tabulação dos questionários do inquérito alimentar da pesquisa. 
SZARFARC, S.C. et al. [A study of children's health in S. Paulo City (Brazil), 1984-1985. X - Food intake]. Rev. Saúde públ., S. Paulo, 22:266-72, 1988.

ABSTRACT: As part of a broad epidemiological survey of the health conditions of under-fives, a random sample $(n=305)$ of children living in S. Paulo city (Brazil) was submitted to a 24-hour dietary recall. The main results were: a) from one year of age, food intake is not sufficiente to cover the energy requirements of a great part of the population; b) among all ages, but particularly in the two first years of life, dietary provision of iron is very far from the recommended requirements; $c$ ) at all ages, protein and vitamin $\mathrm{A}$ intakes are reasonably good. Those findings are consistent with the clinical and biochemical evaluation of nutritional status, conducted simultaneously by the survey. The social stratification of the families revealed that socioeconomic status exerts greater influence in the energy intake than in the iron intake. Different hypothesis were raised to explain the energy and iron deficiencies. In the case of energy, the basic cause seems to be the low purchasing power of the population which determines poor food availability and insufficient intake. In the case of iron, the same hypothesis seems to be applicable only after two years of age. Before this age, factors related to precocious weaning and to delay in the introduction of good sources of iron are apparently more important.

UNITERMS: Child health. Food consumption. Nutrition surveys. Infant nutrition. Infant nutrition disorders. Socioeconomic factors.

\section{REFERÊNCIAS BIBLIOGRÁFICAS}

1. BATISTA FILHO, M. \& BARBOSA, N.P. Pró-memória: alimentação e nutrição 1974/84. Brasilia, Ministério da Saúde. Instituto Nacional de Alimentação e Nutrição, s.d.

2. BROWN, K.H. Clinical and field studies of human lactation methodological considerations. Amer. J. clin. Nutr., 35:745-56, 1982.

3. DEPARTAMENTO INTERSINDICAL DE ESTATISTICA E ESTUDOS SÓCIO-ECONÔMICOS (DIEESE). Nivel alimentar da população trabalhadora da Cidade de São Paulo. Est. sócio-econ., S. Paulo, 1(1) jul., 1973.

4. FAO/WHO/UNU. Expert Consultation on Energy and Protein Requirement, Rome, 1981. Report. Geneva, World Health Organization, 1985. (WHO Techn. Rep. Ser., 724).

5. FEHILY, A.M. Epidemiology for nutritionists: 4 - survey methods. Hum. Nutr. appl. Nutr., 37A:419-25, 1983.

6. FLORES, M. et al. Evaluación dietética de familias y preescolares mediante la aplicacion de diferente métodos y técnicas: area rural de Nicaragua. Arch. lat.amer. Nutr., 23:325-44, 1973.

7. FUNDAÇÃO GETÚLIO VARGAS/ INSTITUTO BRASILEIRO DE ECONOMIA. Food consumption in Brazil: family budget surveys in the early 1960's. Jerusalém, Keter Press, 1970.

8. FUNDAÇĀO IBGE. Consumo alimentar $e$ antropometria. Rio de Janeiro, 1977. (Estudo Nacional de Despesa Familiar, v.1, t.1).

9. FUNDAÇÃO IBGE. Tabela de composiçâo dos alimentos. Rio de Janeiro, 1977. (Estudo Nacional da Despesa Familiar, v. 3 t.1)

10. HAMILL, P.U.U. et al. Physical growth: NCHS percentiles. Amer. J. clin. Nutr., 32:607-29, 1979.

11. INTERNATIONAL NUTRITIONAL ANEMIA CONSULTATIVE GROUP. Iron deficiency in infancy and childhood. Washington, D.C., The Nutritional Foundation, 1979

12. JELLIFFE, D.B. Evaluacion del estado de nutricion de la comunidad: con especial referência a las encuestas en las regiones en desarollo. Ginebra, Organización Mundial de la Salud, 1968. (OMS - Série de Monografias, N. 53.

13. KIRSTEN TIACCHI, J. et al. Pesquisa de orçamentos familiares no Municipio de São Paulo (POF). São
Paulo, Fundação Instituto de Pesquisas Econômicas, 1971/72. (Série de Monografias, v.3).

14. LECHTIG, A. et al. The one-day recall dietary to survey: a review of its usefulness estimate protein and calorie intake. Arch. lat.-amer. Nutr., 26:243-71, 1976.

15. MARTINS, I.S. Requerimentos de energia e nutrientes da população brasileira. Rev. Saúde públ., S. Paulo. 13(supl.1):1-20, 1979.

16. MONTEIRO, C.A. et al. Estudo das condiçôes de saúde das crianças do Municipio de São Paulo, SP (Brasil), 1984/1985. I - Aspectos metodológicos, características sócio-econômicas e ambiente físico. Rev. Saúde públ., S. Paulo, 20:435-45, 1986.

17. MONTEIRO, C.A. et al. Estudo das condiçes de saúde das crianças do Município de São Paulo, SP (Brasil), 1984/1985. II - Antropometria nutricional. Rev. Saúde públ., S. Paulo, 20:446-53, 1986.

18. MONTEIRO, C.A. et al. Estudo das condiçðes de saúde das crianças do Municipio de São Paulo, SP (Brasil), 1984/1985. Ill - Aleitamento materno. Rev. Saúde públ., S. Paulo, 21:13-22, 1987.

19. MONTEIRO, C.A. \& SZARFARC, S.C. Estudo das condições de saúde das crianças do Município de São Paulo, SP (Brasil), 1984/1985. V - Anemia. Rev. Saúde públ., S. Paulo, 21:255-60, 1987

20. MONTEIRO, C.A. \& MEYER, M. Estudo das condiçós de saúde das crianças do Município de São Paulo, SP (Brasil), 1984/1985. VIII - Cobertura e impacto da suplementação alimentar. Rev. Saúde públ., S. Paulo, 22:132-9, 1988.

21. SHRIMPTON, R. Ecologia da desnutrição na infância, análise da evidência das relaçōes entre variáveis sócio-econômicas e estado nutricional. Brasília, Centro Nacional do Recursos Humanos do IPEA/UNICEF, 1986. (Série Instrumentos para Ação, 3).

22. VIACAVA, F. et al. A desnutrição no Brasil; uma análise do Estudo Nacional da Despesa Familiar (IBGE 74/75) para o Nordeste, Estado de São Paulo e Esta. do do Rio de Janeiro. Petrópolis, Ed. Vozes/FlNEP, 1983.

Recebido para publicação em $1 / 10 / 1987$ Reapresentado em 25/4/1988 Aprovado para publicação em 3/5/1988 